\title{
Fully automated sequence alignment methods are comparable to, and much faster than, traditional methods in large data sets: an example with hepatitis B virus
}

Therese A. Catanach corresp., 1,2,3 , Andrew D. Sweet ${ }^{2,4}$ ， Nam D. Nguyen ${ }^{5}$ ， Rhiannon M. Peery ${ }^{6,7}$, Andrew H. Debevec $^{8}$, Andrea K. Thomer ${ }^{9}$, Amanda C. Owings ${ }^{4}$, Bret M. Boyd ${ }^{2,10}$, Aron D. Katz ${ }^{2,11}$, Felipe N. SotoAdames $^{12,13}$, Julie M. Allen ${ }^{2,14}$

1 Ornithology Department, Academy of Natural Sciences of Drexel University, Philadelphia, PA, United States of America

2 Illinois Natural History Survey, University of Illinois at Urbana-Champaign, Champaign, Illinois, United States

3 Department of Wildlife and Fisheries Sciences, Texas A\&M University, College Station, Texas, United States

4 Program in Ecology, Evolution, and Conservation Biology, University of Illinois at Urbana-Champaign, Urbana, Illinois, United States

5 Computer Science and Engineering, University of San Diego, San Diego, California, United States

6 Department of Biology, University of Alberta, Edmonton, Alberta, Canada

7 Department of Plant Biology, University of Illinois at Urbana-Champaign, Champaign, Illinois, United States

8 College of Liberal Arts and Sciences, University of Illinois at Urbana-Champaign, Champaign, Illinois, United States

9 School of Information, University of Michigan - Ann Arbor, Ann Arbor, Michigan, United States

10 Department of Entomology, University of Georga, Athens, Georgia, United States

11 Department of Entomology, University of Illinois at Urbana-Champaign, Champaign, Illinois, United States

12 Florida State Collection of Arthropods, Florida Department of Agriculture and Consumer Services, Gainesville, Florida, United States

13 Department of Entomology and Nematology, University of Florida, Gainesville, Florida, United States

14 Biology Department, University of Nevada - Reno, Reno, Nevada, United States

Corresponding Author: Therese A. Catanach

Email address: tacatanach@drexel.edu

Aligning sequences for phylogenetic analysis (multiple sequence alignment; MSA) is an important, but increasingly computationally expensive step with the recent surge in DNA sequence data. Much of this sequence data is publicly available, but can be extremely fragmentary (i.e. a combination of full genomes and genomic fragments), which can compound the computational issues related to MSA. Traditionally, alignments are produced with automated algorithms and then checked and/or corrected "by eye" prior to phylogenetic inference. However, this manual curation is inefficient at the data scales required of modern phylogenetics and results in alignments that are not reproducible. Recently, methods have been developed for fully automating alignments of large data sets, but it is unclear if these methods produce alignments that result in compatible phylogenies when compared to more traditional alignment approaches that combined automated and manual methods. Here we use approximately 33,000 publicly available sequences from the hepatitis B virus (HBV), a globally distributed and rapidly evolving virus, to compare different alignment approaches. Using one data set comprised exclusively of whole genomes and a second that also included highly fragmentary 
sequences, we compared three MSA methods: 1) a purely automated approach using traditional software, 2 ) an automated approach including by eye manual editing, and 3 ) more recent fully automated approaches. To understand how these methods affect phylogenetic results, we compared resulting tree topologies based on these different alignment methods using multiple metrics. We further determined if the monophyly of existing HBV genotypes was supported in phylogenies estimated from each alignment type, and under different statistical support thresholds. Traditional and fully automated alignments produced similar HBV phylogenies. Although there was variability between branch support thresholds, allowing lower support thresholds tended to result in more differences among trees. Therefore differences between the trees could be best explained by phylogenetic uncertainty unrelated to the MSA method used. Nevertheless, automated alignment approaches did not require human intervention and were therefore considerably less time-intensive than traditional approaches. Because of this, we conclude that fully automated algorithms for MSA are fully compatible with older methods even in extremely difficult to align data sets. Additionally, we found that most HBV diagnostic genotypes did not correspond to evolutionarily sound groups, regardless of alignment type and support threshold. This suggests there may be errors in genotype classification in the database or that HBV genotypes may need a revision. 
1 Fully automated sequence alignment methods are

2 comparable to, and much faster than, traditional

3 methods in large data sets: an example with hepatitis B

4 virus

5

6 Therese A. Catanach ${ }^{*}$ Corresp., 1,2,3, Andrew D. Sweet ${ }^{* 2,4}$, Nam D. Nguyen ${ }^{5}$, Rhiannon M. Peery ${ }^{6,7}$,

7 Andrew H. Debevec ${ }^{8}$, Andrea K. Thomer ${ }^{9}$, Amanda C. Owings ${ }^{4}$, Bret M. Boyd ${ }^{2,10}$, Aron D.

8 Katz $^{2,11}$, Felipe N. Soto-Adames ${ }^{12,13}$, Julie M. Allen ${ }^{2,14}$

$9{ }^{1}$ Ornithology Department, Academy of Natural Sciences of Drexel University, Philadelphia, PA, 10 United States of America

${ }^{2}$ Illinois Natural History Survey, University of Illinois at Urbana-Champaign, Champaign, Illinois, United States

${ }^{3}$ Department of Wildlife and Fisheries Sciences, Texas A\&M University, College Station, Texas, United States

${ }^{4}$ Program in Ecology, Evolution, and Conservation Biology, University of Illinois at UrbanaChampaign, Urbana, Illinois, United States

${ }^{5}$ Computer Science and Engineering, University of San Diego, San Diego, California, United States

${ }^{6}$ Department of Biology, University of Alberta, Edmonton, Alberta, Canada

${ }^{7}$ Department of Plant Biology, University of Illinois at Urbana-Champaign, Champaign, Illinois, United States

${ }^{8}$ College of Liberal Arts and Sciences, University of Illinois at Urbana-Champaign, Champaign, Illinois, United States

${ }^{9}$ School of Information, University of Michigan - Ann Arbor, Ann Arbor, Michigan, United States

${ }^{10}$ Department of Entomology, University of Georgia, Athens, Georgia, United States

${ }^{11}$ Department of Entomology, University of Illinois at Urbana-Champaign, Champaign, Illinois, United States

${ }^{12}$ Florida State Collection of Arthropods, Florida Department of Agriculture and Consumer Services, Gainesville, Florida, United States 
$31{ }^{13}$ Department of Entomology and Nematology, University of Florida, Gainesville, Florida, 32 United States

$33{ }^{14}$ Biology Department, University of Nevada, Reno, Nevada, United States

34 Corresponding Author: Therese A. Catanach

35 Email address: tacatanach@drexel.edu

* Denotes co-first authors

\section{Abstract}

41

Aligning sequences for phylogenetic analysis (multiple sequence alignment; MSA) is an important, but increasingly computationally expensive step with the recent surge in DNA sequence data. Much of this sequence data is publicly available, but can be extremely fragmentary (i.e. a combination of full genomes and genomic fragments), which can compound the computational issues related to MSA. Traditionally, alignments are produced with automated algorithms and then checked and/or corrected "by eye" prior to phylogenetic inference. However, this manual curation is inefficient at the data scales required of modern phylogenetics and results in alignments that are not reproducible. Recently, methods have been developed for fully automating alignments of large data sets, but it is unclear if these methods produce alignments that result in compatible phylogenies when compared to more traditional alignment approaches that combined automated and manual methods. Here we use approximately 33,000 publicly available sequences from the hepatitis B virus (HBV), a globally distributed and rapidly evolving virus, to compare different alignment approaches. Using one data set comprised exclusively of whole genomes and a second that also included highly fragmentary sequences, we compared three MSA methods: 1) a purely automated approach using traditional software, 2) an automated approach including by eye manual editing, and 3) more recent fully automated 
58 approaches. To understand how these methods affect phylogenetic results, we compared

59 resulting tree topologies based on these different alignment methods using multiple metrics. We

60 further determined if the monophyly of existing HBV genotypes was supported in phylogenies

61 estimated from each alignment type, and under different statistical support thresholds.

62 Traditional and fully automated alignments produced similar HBV phylogenies. Although there

63 was variability between branch support thresholds, allowing lower support thresholds tended to

64 result in more differences among trees. Therefore differences between the trees could be best

65 explained by phylogenetic uncertainty unrelated to the MSA method used. Nevertheless,

66 automated alignment approaches did not require human intervention and were therefore

67 considerably less time-intensive than traditional approaches. Because of this, we conclude that

68 fully automated algorithms for MSA are fully compatible with older methods even in extremely

difficult to align data sets. Additionally, we found that most HBV diagnostic genotypes did not

correspond to evolutionarily sound groups, regardless of alignment type and support threshold.

71 This suggests there may be errors in genotype classification in the database or that HBV

72

73

74

75

76

77

78

79

80

81

82

83

84

85 genotypes may need a revision.

4

6




\section{Introduction}

88

89

90

91

92

93

94

95

96

97

The multiple sequence alignment (MSA) is arguably one of the most important steps in a phylogenetic analysis (Morrison, 2006; Kemena and Notredame, 2009), but can be difficult to perform accurately on large data sets (Liu et al., 2010). Traditionally, MSA methods for phylogenetic reconstruction are performed using alignment programs such as MUSCLE (Edgar, 2004) followed by manual, "by eye" corrections (Hillis et al., 1996; Hall, 2001; Edgar and Batzoglou, 2006). Studies such as the one by Kjer et al. (2007) found that manual curation of MSA data was common in studies published in journals focused on phylogenetics. Google Scholar searches including terminology associated with manual editing of MSA reveals tens of thousands of hits (search completed March 21, 2018). Similarly, Morrison (2009) surveyed 247 systematics papers published in 2007 and found that $76 \%$ of these studies included a manual curation step. Therefore, while only mentioned in passing, manual editing of MSA is a common practice. However, as data sets have grown larger, manual examination of each alignment has become increasingly difficult. Furthermore, the results of such effort cannot be replicated and may lead to inconsistencies between studies. These differences may be significant in data sets where manual editing is especially challenging; for example, data sets with many taxa $(>10,000)$ and varying sequence lengths. This problem can become increasingly difficult if there are repeat elements in the genome and rapidly evolving sites, like those seen in viruses. Although methods have recently been developed for fully automating alignments with large data sets, (e.g. PASTA; Mirarab et al., 2014) and for adding in fragmentary sequences to longer stretches of DNA (e.g. MAFFT --addfragments; Katoh et al., 2002; Katoh and Martin, 2012, UPP; Nguyen et al., 2015), 
109 it is unclear if these fully automated methods result in equivalent alignments (i.e. result in trees

110 with similar topologies) when compared to an alignment created using the traditional approach.

111 In recent decades, molecular phylogenetics has experienced a boom due to the generation

112 of increasingly larger DNA sequence data sets for addressing difficult biological questions

113 (Eisen and Fraser, 2003; Delsuc et al., 2005; Philippe et al., 2005; Jarvis, 2016). This increase in

114 data has resulted in many phylogenetic studies with thousands of taxa and characters (e.g.

115 thousands of genes, transcriptomes, etc.; Kozlov, 2015; Arbizu, 2014; Jarvis, 2014; Misof, 2014;

116 Faircloth et al., 2013; Heyduk et al., 2015; Leache and Linkem, 2015; Harkins, 2016; Yang and

117 Qiu, 2016). While larger data sets may help resolve difficult phylogenetic questions, they also

118 present computational challenges (Sanderson and Driskell, 2003; Soltis et al., 2007). A

119 byproduct of the increased DNA sequencing efforts is the large amount of publicly available

120 sequences in databases including GenBank, Ensemble, and DDBJ (Benson et al., 2014; Yates et

121 al., 2016; Mashima et al., 2016). Although the number of whole genome sequences in these

122 databases will continue to increase with the widespread adoption of next generation sequencing

123 (NGS) technology, currently a considerable portion of available data are genomic fragments (e.g.

124 from Sanger sequencing; [Benson et al., 2014; http://www.ncbi.nlm.nih.gov/genbank/statistics/]).

Viruses can pose unique challenges for MSA and phylogenetic inference. These

organisms often have relatively small genomes and undergo rapid evolution, making it difficult

127 to assess homology within alignments (Beerenwinkel et al., 2012; Nasir and Caetano-Anolles

128 2015). However, due to their medical relevance, viruses are well-represented in public sequence

129 databases (Chooka et al., 2015; https://www.ncbi.nlm.nih.gov/genome/viruses/) as a mix of full

130 genomes and sequence fragments. In most cases, available sequences from these taxa are from

131 regions of the genome selected for diagnostic purposes (Weber, 2005). These areas tend to be 
132 fast evolving and short in length (Osiowy et al., 2006). Incorporation of these sequences in

133 phylogenetic analyses is useful, but integrating them into a MSA with full genome sequences can

134 be challenging. Global alignments find similarities between sequences from beginning to end

135 and local alignments try to find a similar region to align from in all sequences (Philippe et al.,

136 2011; Nguyen et al., 2015), thus, both approaches fail completely when sequences have no

137 characters in common. New approaches that are a hybrid of alignment methods, an increasingly

138 active research area, are appropriate for data with mixed fragment and genome information

139 (Schmollinger et al., 2004; Subramanian et al., 2005; Liu et al., 2012; Hossain et al., 2013; Ye et

140 al., 2015; Nguyen et al., 2015).

Here we focus on the hepatitis B virus (HBV), a medically important and globally

142 distributed virus (Okamoto et al., 1988; Norder, Courouce, \& Magnius, 1994; Stuyver et al.,

143 2000; Arauz-Ruiz et al., 2002; Simmonds \& Midgley, 2005; Tran, Trinh, \& Abe, 2008;

144 Tatematsu et al., 2009; Kurbanov, Tanaka, \& Mizokami, 2010). Every year ca. 750,000 people

145 die from the virus (Lozano et al., 2013) and in some geographic regions up to $20 \%$ of the adult

146 population have chronic HBV (Chen, 1993; Liaw \& Chu, 2009). There are currently 10

147 recognized genotypes of HBV designated A-J. These genotypes are primarily associated with

148 particular geographic regions, but are also identified based on genetic divergence (Hernandez et

149 al., 2014). A push to understand the global distribution and diversity of the virus (Shi et al.,

150 2013), along with associated global health implications (Shi, 2012), has resulted in large amounts

151 of publicly available HBV sequence data. The S gene region (barcoding region of the HBV

152 genome; Galibert et al., 1979), is the most abundant HBV sequence in GenBank. However, many

153 full genome sequences are also available (Wu et al., 2008). 
HBV has a compact circular genome $\sim 3.2 \mathrm{~kb}$ in length, with four genes arranged across

155

156

157

158

159

160

161

162

163

164

165

166

167

168

169

170

171

172

173

174

175

176

177

overlapping reading frames (Fig. 1; Norder et al., 1994). Any attempt to use publicly available HBV sequence data for phylogenetic analysis needs to work with the fragmentary nature of the data set and with the issues of limited character sampling (i.e. small genomes). The small size of the genomes coupled with the large number of sequences creates phylogenetic data sets that are "tall and narrow". Furthermore, there is currently no universally agreed starting position for viruses with circular genomes, including HBV, therefore individual researchers are free to report genome sequences starting at any position, and genomes may have arbitrary breakpoints. This results in sequence alignment issues as homologous portions of the genome are not necessarily in the same relative position when represented as linear sequences.

To ameliorate these issues in virus MSA, we compared traditional automated-manual alignment approaches to fully automated methods for phylogenetic analyses on a tall and narrow data set generated from publicly available HBV sequence data. Using two different data sets, one comprised exclusively of whole genomes (and therefore with limited missing data) and a second comprised of most publicly available HBV sequences (a highly fragmentary data set) we compared three alignment methods: 1) traditional automated MSA methods , 2) the commonly used hybrid approach of MSA followed by manual adjustment of the alignment, and 3) UPP and PASTA, two modern automated MSA methods. This approach allowed us to assess the effectiveness of different methods for handling the problem of large matrices with highly fragmentary sequence data. Additionally, through MSA evaluation methods we were able to assess the phylogenetic signal of and monophyly within named strains of the virus.

\section{Methods}

\section{Data Downloads and Initial Cleaning}


179 hepatitis B sequences using the NCBI Taxonomy Browser (Taxid: 10407) on March 20, 2013.

180 This included both full genome and fragmentary sequence data. This initial download yielded

18155,353 sequences. Sequences from non-primate hosts, less than $100 \mathrm{bp}$ in length, lab strains, and

182 recombinant genomes were removed.

183 Sequence Alignment

184 Here we detail our approaches for aligning genomes only and all (genomes + fragmentary)

185 HBV sequence data sets (Table 1). The workflows for both approaches are summarized in Fig. 2

186 and a more detailed version can be found in Supplementary Fig. 1. Commands for relevant

187 programs are listed in the supplementary information (see Supplementary Information on Dryad, 188 DOI: https://doi.org/10.5061/dryad.nc220).

189

190

191

192

\section{Genome Alignments}

\section{Traditional alignment}

To generate the genomes-only data set, we selected whole genomes from our initial filtered GenBank download. Whole genomes were identified by sequence length. The HBV genome is estimated to be ca. $3.2 \mathrm{~kb}$ long, therefore we selected sequences $\geq 3,000$ bp to be included in our full genome data set. In total, we identified 5,553 full genome sequences.

There is not a universal starting point within the HBV genome, necessitating the development of a customized script to "linearize" the HBV sequences for the alignment (https://github.com/tacatanach/Hepatitis_B_Virus_alignment). This script chose a universal starting point in the alignments, and removed any overhang from the end and placed it at the beginning. The "linearized" sequences were then aligned using the default gap parameters in MUSCLE (Edgar, 2004) and manually adjusted using SeaView v.4 (Gouy, Guindon, \& Gascuel, 2010). We denote this alignment as the "MUSCLE-manual alignment". In addition, we aligned 
203 the genomes without manual adjustment using MUSCLE, Clustal-Omega, MAFFT, and PASTA

204 (see Table 2 for version information). However, we primarily focused on the MUSCLE-manual

205 alignment to be comparable with analyses using the total data set (see below). Sequences that

206 were not alignable because they were either too taxonomically divergent, had reported

207 recombination between strains in the GenBank information, or had evidence of unreported

208 recombination were removed. Unreported recombination was inferred when a region did not

209 align well and BLAST results indicated that the region was a strong match for a different region

210 of the reference genome. These recombinants can be the result of both inter- and intra-strain

211 recombination of the genome. This alignment was manually checked by three authors.

212

As a final quality check of the MUSCLE-manual alignment, we inferred a neighbor-

213 joining (NJ) tree using the R package ape (Paradis et al., 2004). In order to remove poorly

214 aligned sequences, we iteratively ran NJ quality control steps (NJ QC; available at

215 https://github.com/tacatanach/Hepatitis_B_Virus_alignment) as follows. We flagged sequences

216 with outlier branch lengths (branches whose leaf to the nearest node distance were $>1.5$ times

217 the interquartile range of the other branch lengths), and removed these outliers from the

218 alignment. We then repeated the NJ QC step two more times to remove any remaining poorly

219 aligned sequences. The final alignment included 5,244 sequences.

220

221

222

223

224

225

226

\section{Automated alignment}

To compare the traditional method of MSA (using alignment software followed by manual adjustment) with fully automated approaches, we started with the 5,244 individual sequences identified in the manual method as high quality genomes. We then aligned these sequences with several alignment methods without manually adjusting the resulting alignment. The input data were linearized and degapped prior to alignment. For alignment software we used 
227 PASTA v.1.6.3 (Mirarab et al., 2015), MAFFT v7.305b (Katoh and Standley 2013), Clustal

228 Omega v1.2.4 (Sievers et al. 2011), and Muscle v3.8.31 (Edgar 2004). In addition, we used

229 PASTA to align the original 5,244 (i.e. un-linearized) genome sequences.

230

231

232

233

234

235

236

237

238

239

240

241

242

243

244

245

246

247

248

249

250

\section{Total (Fragments + Genome) alignments}

\section{Traditional Alignment}

A traditional, brute force approach to align all 55,353 sequences (including full genomes and fragmentary sequences) was not feasible due to the large number of sequences and alignment gaps from the fragments. Therefore, to align the full set of sequences we first used a custom script to group sequences into 1,269 FASTA files based on their GenBank accession numbers (https://github.com/tacatanach/Hepatitis_B_Virus_alignment). Because GenBank accession numbers are assigned sequentially at submission, a cluster of successive HBV sequences is likely from the same region of the genome. As with the genome sequences, we used our custom script to "linearize" the original GenBank sequences. We also created a consensus sequence of the full HBV genome $(3,639 \mathrm{bp})$ based on the genome alignment, using majority rule (rather than ambiguity codes) for ambiguous sites. This consensus sequence served as a reference for downstream alignment steps. Each author then aligned about 150 files, using the default gap parameters in MUSCLE and checking each alignment by eye against the genome consensus sequence. To facilitate aligning by eye we used custom scripts to "ladderize" the alignments by ordering the sequences according to starting position and length (https://github.com/tacatanach/Hepatitis_B_Virus_alignment) so that in each alignment sequences with similar starting points and lengths were placed next to each other. To combine two alignments we used profile-profile in MUSCLE followed by manual adjustments against the genome consensus. We iteratively repeated this process to gradually combine the sequences. 
251 This process was successful for several rounds of profile-profile alignments. However, the

252 alignment files eventually became too large for the profile-profile function in MUSCLE to

253 consistently align pairs of files. At this point, we began manually combining pairs of aligned

254 sequence files using the cat Unix command followed by manual adjustments. Adjustments were

255 made by opening gaps in each alignment subset to align with the reference.

256 Additionally, there were 930 individually uploaded sequences ("singletons"; i.e.

257 sequences that did not cluster with other sequences according to accession number). To align

258 these sequences with the larger sequence matrix, we first split all singletons into files of 50-100

259 sequences, each file containing a consensus genome sequence. These sequences were then

260 aligned by eye to the consensus genome sequence, and resulting aligned sequences files were

261 combined as described above. We then manually combined all aligned sequence files as

262 described above, using the genome consensus sequence as a reference.

263 To further check the alignment, we created consensus sequences (with ambiguity codes)

264 for blocks of 73 (the number of lines viewable in a screen without scrolling; for ease of viewing)

265 sequences (https://github.com/tacatanach/Hepatitis_B_Virus_alignment). We then combined all

266 consensus sequences maintaining the alignment structure. By collapsing sets of sequences into a

267 single consensus sequence we could visually check the entire alignment. If consensus sequences

268 were not aligned to each other or contained many ambiguity codes, this indicated a particular

269 portion of the alignment was misaligned. We then adjusted the corresponding alignment

270 accordingly and repeated the process until we could not detect any misaligned regions.

271 During the alignment step, 5,000 sequences could not be aligned because they were

272 unflagged recombinants or from non-humans hosts and were subsequently removed. We also ran

273 five iterations of the NJ QC step and removed an additional 168 sequences. Finally, we checked 
274 to ensure that sequences from unique hosts, geographic regions, ancient (more than 100 years

275 old) samples, and all genotypes were present in the alignment. If any of these key data were

276 removed through prior filtering steps, they were manually added back into the alignment.

277 Including these sequences is important for having a representative sample, and warranted the

278 additional effort required to incorporate them into our alignment. We also included a sequence of

279 HBV from woolly monkey (GenBank accession JX978431) as an outgroup.

280

281

282

283

284

286

287

288

289

290

291

292

293

294

295

296

297

\section{Automated alignment}

In order to obtain an automated alignment of the total data set, we used UPP v.3.0

(Nguyen et al., 2015), a method designed for inserting fragmentary sequences into an existing backbone alignment using a backbone tree to guide the process. To evaluate the influence a backbone tree may have on UPP alignments, we ran a pair of UPP analyses, one with a tree from the PASTA genomes-only alignment as a backbone and a second using a tree from the Musclemanual genomes-only alignment. We estimated Maximum Likelihood (ML) backbone trees under GTR+CAT using FastTree-2 v.2.1.7 (Price, Dehal, \& Arkin, 2010) on the backbone alignments. We selected PASTA as the automated alignment to compare against the Musclemanual alignment because PASTA uses MAFFT internally and results in a similar backbone tree topology as the Clustal-Omega and MAFFT alignments (Supplementary Fig. 2).

\section{S-region}

The HBV S-region is often sequenced for identification of viral genotype (Galibert et al., 1979). Therefore, as a comparison to the genomes-only and total (genomes + fragments) data sets, we identified and analyzed the S-region in the whole manual genome alignment based on the annotation of GenBank accession AJ131956 (Ozaslan et al., 2007). The annotation of the Sregion combines pre-S1, pre-S2, and the $\mathrm{S}$ gene. 


\section{Tree Estimation}

300

For each data set we inferred a ML phylogeny with bootstrap support. First, we generated

301

302

303

304

305

306

307

308

309

310

311

312

313

314

315

316

317

318

319

320

100 bootstrap replicate alignments in RAxML v.8.2.10 (Stamatakis, 2010). For each bootstrap

replicate we estimated an ML tree using FastTree2. We then used FastTree to estimate a best ML tree from the original alignment, and summarized the bootstrap replicates on this tree using SumTrees v. 4.0.0 in DendroPy (Sukumaran \& Holder, 2010).

\section{Tree Comparisons}

To compare the similarity of tree topologies, we did pairwise comparisons of the trees produced using each of the MSA strategies. For each of the trees we collapsed nodes based on three bootstrap support thresholds $(50 \%, 75 \%, 90 \%)$. We then calculated the total number of edges for each tree and the number of edges that were incompatible with the other tree in the pairwise comparison using Phylonet Modified (Mirarab et al., 2014). We also calculated Robinson-Foulds (RF) distances (Robinson \& Foulds, 1981) for each tree comparison using CompareTree.pl (Fast Tree-Comparison Tools, 2009). However, due to how RF distances are calculated, comparing this metric across trees can be misleading. For example, two clades which are identical in topology except for the placement of one taxon can have a high RF value if the one non-identical node is placed at the tip of a clade in one tree but at the base in the second tree. Therefore, although we include the RF metric, we interpret our results based primarily on the incompatibility ratios. We performed all possible pairwise comparisons between the different trees for a specific support cutoff. We then converted each comparison to a ratio of the number of incompatible edges to the total number of edges in the comparison. For comparisons between 
321 genome and total alignment trees, we pruned the total alignment trees to be the same set of taxa 322 as the genome-only alignments.

As a way to assess tree differences in a biologically meaningful context, we mapped genotype labels onto our tree tips. We pruned out tips that did not have any associated genotype data or were recombinant (i.e. were associated with multiple genotypes) based on GenBank metadata. For each genotype, we then identified the smallest possible clade that included all taxa

327 of the genotype, and calculated the percentage of tips in the clade identified as that genotype using a python script mono.py (https://github.com/tacatanach/Hepatitis_B_Virus_alignment). We call this metric the "genotype occupancy proportion." We repeated this analysis for each bootstrap collapse threshold $(50 \%, 75 \%$, and $90 \%)$ of each tree.

\section{Data sets}

\section{Full Genomes and S-Region}

The initial genomes-only alignment was 7,069 characters long. Quality trimming steps for the genomes-only data set removed 309 sequences. "Linearizing" and manually aligning the trimmed subset resulted in a sequence matrix with 5,244 taxa and 4,269 characters. The MUSCLE alignment with the same sequences resulted in an alignment with 4,661 characters, and the PASTA alignment was 3,423 characters long. The S-region-only alignment from the manual genomes alignment was 1,340 characters long. All three alignments reveal similar Dryad), with a mean p-distance of 0.08 and max p-distance of 0.20 .

\section{Total data set}


All sequences included in the genome-only alignment were also included in the total

347 alignment. After filtering the initial NCBI download, we had a data set of 37,788 sequences,

348 including both full genome and fragmentary sequences (Supplementary Table 1 on Dryad). After

349 iteratively aligning sequence files and filtering out divergent sequences (recombinants, etc.), the

350 final alignment included 32,819 sequences. "Linearizing" the full alignment and removing gap-

351 only columns resulted in a final alignment length of 5,196 characters. This same data set was

352 used to create two UPP alignments: 1) using the manually-aligned genomes only data set as a

353 backbone (final alignment: 4,205 characters); and 2) using a PASTA-aligned backbone (final

354 alignment: 3,387 characters).

355

356

357

358

359

360

361

362

363

364

365

366

367

368

\section{Tree comparisons}

Comparisons between trees from the different alignment types showed different levels of compatibility depending on bootstrap cutoff and alignment type, and are summarized in Figs. 3 and 4. Specific values are listed in Supplementary Table 1 (on Dryad). Trees without any edges collapsed ( $0 \%$ threshold) had the highest incompatibility ratios. The lowest ratio values for a $0 \%$ threshold were from comparisons between genome alignment trees, but these were still considerably higher than ratio values from comparisons at all other thresholds. Incompatibility ratios tended to decrease with an increasing bootstrap threshold. The lowest ratio values were for comparisons at the $75 \%$ and $90 \%$ thresholds. Ratios for comparisons at the $50 \%$ threshold tended to be higher than those for comparisons at the $75 \%$ and $90 \%$ thresholds, but were all considerably lower than comparisons at the $0 \%$ threshold. RF values also tended to be higher for comparisons at the $0 \%$ threshold, but this was not true across all comparisons. The lowest RF values were from comparisons at higher thresholds. However, there was not a clear pattern 
369

370

371

372

373

374

375

376

377

378

379

380

381

382

383

384

385

386

387

388

389

390

391

among comparisons at the $50 \%, 75 \%$, and $90 \%$ thresholds. For example, several $90 \%$ threshold comparisons had higher RF values than some $50 \%$ and $75 \%$ threshold comparisons.

Among trees from the different alignment types, trees from the S-region alignment tended to have higher incompatibility ratio values. This was true at each bootstrap threshold. Comparisons including genome alignment trees tended to have the lowest ratios whereas the highest values involved comparisons between the genome and total alignments. Trees from total (genomes + fragments) alignments had intermediate ratios. Trees from the total automated alignments (with UPP) had slightly lower ratios than the total manual alignment, but the two UPP trees had the fewest number of differences between them (Fig. 4, Supplementary Table S1 on Dryad). For the RF metric, the lowest values came from comparisons among major alignment types. Genome-genome comparisons and total-total comparisons had the lowest RF values. Trees from the S-region alignment had similar RF values across comparisons with other trees, except for comparisons at the $0 \%$ threshold.

The average absolute difference in genotype occupancy proportions between the different alignments was $4.6 \%$ for genomes and S-region trees, and $2.1 \%$ for total alignment trees. Overall, the average proportions of genotype occupancy across all alignment types, genotypes, and support thresholds were $52 \%$ for genome and S-region trees, and $13.1 \%$ for total alignment trees. These patterns are summarized in Fig. 5, and specific genotype occupancy proportion values are listed in Supplementary Table S2 (on Dryad). However, there was a great deal of variability between genotypes. Genotypes A-C, G, and genotype I consistently exhibited clade occupancy values between 15\%-38\% among the different alignment types (genomes, Sregion, total) and bootstrap support collapse cut offs. Conversely, genotypes E, F, and H consistently accounted for most (85\%-100\%) of the tips in their respective clades in genome and 
392 S-region trees, but lower than $10 \%$ in the total alignment trees. Although these genotypes are

393 relatively rare in GenBank (150 or fewer genome sequence and 530 or fewer total sequences per

394 genotype), they are geographically widespread (Shi et al. 2013). The addition of fragmentary

395 sequence data could have greatly increased the geographic coverage of the data set which could

396 result in an increase in genetic variation within the genotype and an extreme decrease in

397 genotype occupancy. Additionally, as we were limited to GenBank annotations for identifying

398 genotype, it is possible that the inclusion of the fragmentary data, an inclusion that in some

399 instances increased the number of sequences six-fold, resulted in introducing sequences that had

400 been incorrectly annotated.

401

Genotype D showed more complex patterns than the other genotypes. On one hand,

402 genotype D accounted for a high proportion (99\%) of tips in the " $\mathrm{D}$ " clade for most genome

403 alignment types and support thresholds. However, the S-region only illustrated a high proportion

404 of genotype occupancy at the $0 \%$ bootstrap cutoff. Additionally, at the $90 \%$ bootstrap cut off

405 only the manual genome alignment had a high occupancy value, whereas the other two genome

406 alignments (MUSCLE and PASTA) had a value of 22\%. This lower value was similar to

407 occupancy proportion values in all total alignment categories for genotype D.

408

409

410

411

412

413

414

\section{Labor requirements}

We compare the labor requirements for the automated alignment methods (MUSCLE, Clustal Omega, MAFFT, PASTA) with the fully manual MUSCLE-manual alignment. All automated alignments were run on a dedicated machine with 24 nodes. Automated methods that were parallelized completed quickly, with MAFFT requiring 4 minutes of wall clock time, Clustal Omega 2.25 hours, and PASTA requiring 3.5 hours. MUSCLE, on the other hand, is a single threaded program and required 32 hours to align. An additional 72 person-hours were needed to 
415 manually hand-curate the MUSCLE alignment to produce the MUSCLE-manual alignment. It is

416 important to note that both PASTA and UPP can result in slightly differing alignments over

417 multiple runs due to the use of multiple cores.

418 An additional 36 hours were needed to align the total data set (27,575 fragments plus

419 whole genomes) using UPP and the genome backbones (from PASTA and the manual

420 alignment). Although an exact estimate of the manual alignment of this same data set is not

421 possible, in comparison the process took $>1,000$ person-hours divided between nine authors over

422 a two year period.

423

424

425

426

427

428

429

430

431

432

433

434

435

436

437

438

\section{Discussion}

Comparisons among phylogenetic trees of HBV indicated that sequence alignment strategy did not have a major effect on tree topology. Within the data set containing only complete genomes, all alignment methods (ClustalOmega, PASTA, MAFFT, MUSCLE, MUSCLE with manual adjustments, or completely manual) produced phylogenies without noticeable differences using three methods of evaluation (clade occupancy, RF values, and incompatibility ratios). Additionally, when two of these whole genome alignments (PASTA and manual alignments) served as a backbone to align genome fragments with UPP, the resulting phylogenies were not different in any biologically meaningful way based on our metrics.

When considering support values for phylogenetic trees produced by different alignment methods, trees with a minimum acceptable bootstrap support threshold of $50 \%$ or greater were compatible (less than $20 \%$ of edges were incompatible). These results suggest multiple alignment approaches are effective for highly fragmented data sets. However, because manual editing of large fragmentary data sets is laborious, the fully automated approach using 
439 appropriate software (e.g., UPP or MAFFT --addfragments) to align these types of data sets is

440 preferred. The traditional method of checking automated alignments does consume time that

441 could be devoted to other tasks, can be difficult to replicate, and is impractical for large data sets.

442 Yet, accuracy remains the goal of phylogenetic estimation and is dependent on the alignment

443 step and is the driver for retention of manual curation. Here we have demonstrated that manual

444 alignment and curation give comparable results to automated methods. This is encouraging, 445 given that publically available data from high-throughput NGS sequencing projects is increasing

446 exponentially and will likely drive a greater reliance on automated methods. It also suggests that 447 findings based on completely automated methods are comparable to results from earlier studies

448 that may have included a manual step. Integrating complete data with existing fragmentary

449 sequences is crucial for addressing biologically relevant questions and in our data, we have

450 shown this can be done so in a consistent manner across commonly used methodologies.

$451 \quad$ Although minor differences in tree topologies are expected when multiple analytical

452 approaches are used, these differences may not be biologically meaningful (i.e rearrangement of

453 branches within a clade). To test the impact of alignment type on the biological meaning of the

454 resulting phylogenies we used HBV genotypes, a feature often annotated by researchers when

455 submitting HBV sequences to GenBank. We sought to determine if HBV genotypes where stable

456 in their location in the tree (consistent) and if they were monophyletic (accurate with regard to

457 evolutionary relatedness) within our tree. Our results are consistent that the genotype occupancy

458 in a clade remain stable across alignment types for both genome and fragmentary data sets. This

459 indicates the alignment approach for these types of data sets does not have a major effect on the

460 biological interpretation of the inferred topologies, particularly when testing the monophyly of a

461 particular subset of tips. This is consistent with our test of incompatible edges among topologies 
462 when considering bootstrap support. This assessment also supports using the fully automated

463 approaches, because using considerably more time to manually adjust alignments does not

464 appear to result in different biological interpretations.

465 Despite genotypes exhibiting consistent clade composition across the different alignment

466 approaches tested, the genome and fragmentary topologies generally had very different

467 occupancy values between them. Topologies from the genome alignments tended to have higher 468 genotype occupancy than topologies from the fragmentary alignments. However, neither data set 469 consistently showed high occupancy. The genotypes that did have good occupancy values (> $47085 \%)$ were often represented by few tips $(<100)$. This pattern of low occupancy was often consistent across support thresholds, suggesting that tree estimation error is not a primary source

472 of the non-monophyly of genotypes. Therefore, most HBV genotypes appear to be paraphyletic

473 or polyphyletic and do not reflect natural groups. The ten HBV genotypes are generally

474 delimited geographically (Stuyver et al., 2000), but are also determined based on sequence 475 divergence (Hernandez et al., 2014). The S-region, which encodes the surface antigen of the 476 virus, is often used as a barcoding region to identify samples to genotypes (Fig. 1; Stuyver et al., 477 2000). In our analysis, the topology from the S-region had similar genotype occupancy values to 478 other full genome alignment topologies, but the values were still low on average. However, the D 479 genotype exhibited notable discrepancies in occupancy values between the S-region and genome 480 alignments, particularly at higher bootstrap collapse thresholds. Additionally, the S-region 481 topology had the highest ratios of incompatible edges with other topologies, regardless of 482 support threshold cutoff. Convergent molecular evolution or horizontal gene transfer could 483 potentially account for discrepancies among trees and genotypes, although our data set is not 484 ideal for demonstrating these mechanisms. Regardless, our results suggest the S-region is not 
485 ideal as a basis for phylogenetic interpretation, and that there is a conflict in phylogenetic signal 486 between the S-region and the rest of the genome.

Our HBV DNA alignment is "tall and narrow," characterized by having many individual

488

489

490

491

492

493

494

495

496

497

498

499

500

501

502

503

504

505

506 sequences but relatively few characters. In this instance, the number of sequences in the

alignment matrix (height) is much greater than the number of characters (length), particularly for the data set including both genomes and fragments. This total data set has 32,819 sequences

(height) and each contained at most 5,196 characters (width) including gap characters. One way to improve phylogenetic resolution of "tall and narrow" data sets is to increase the width of the alignment matrix by adding characters (Rokas et al., 2003; Rokas \& Carroll, 2005; Hedtke et al., 2006; Heath et al., 2008). However, this may not be possible for organisms with small genomes. HBV has a genome size of only approximately $3.2 \mathrm{kbp}$, and our alignments represent the maximum possible number of characters available. These types of data sets are only going to increase in height. Many of these sequences are also likely to be fragments of the full genome, particularly if data is added from publicly available databases. These characteristics can render traditional alignment approaches unfeasible. Furthermore, many of the organisms that are wellrepresented in public sequence databases and tend to have smaller genomes are likely to be medically important viruses and bacteria. Accurate phylogenetic estimation of these organisms is necessary for properly understanding their global diversity patterns, a crucial part of epidemiological studies. The results from our study with HBV show that "tall and narrow" data sets can be rapidly aligned using available software, and that the resulting phylogenies are comparable to phylogenies estimated from sequences aligned with a traditional approach. There were also no substantial differences of biological relevance, in this case monophyly of HBV 
507 genotypes. Together these results suggest the rapid, automated approach will be useful for other

508 "tall and narrow" data sets, including those of medically important taxa.

509 Another issue facing phylogenetic estimation of HBV is the lack of a universal sequence

510 starting point. Although the genomes are circular, sequences are uploaded to GenBank in linear

511 format. Without a universal starting location, publicly available HBV sequences begin at

512 different places in the genome. Additionally, the 3' end of one sequence may be orthologous to

513 the 5' end of another sequence. In a sequence alignment, the two sequences would only overlap

514 over a fraction of their entire length, and the whole alignment would be much longer than

515 necessary. Indeed, when we initially used PASTA to align HBV genomes downloaded from

516 GenBank, the alignment was approximately 19,000 bp in length, or 6 times longer than the

517 length of the HBV genome. It was therefore necessary to "linearize" the alignments by moving

518 3' sequences to the 5 ' end before aligning the sequences using a fully automated approach. A

519 similar approach should be taken by future studies focusing on HBV phylogenetics, or for other

520 organisms with similar data issues. Our script chooses an arbitrary cutoff point for

521 "linearization," but perhaps future approaches should choose alignment starting points based on

522 genes or other biologically relevant information. Databases could also implement a standardized

523 starting point for data deposited from these circular genomes.

\section{Conclusion}

Here we demonstrate that UPP and PASTA, completely automated MSA approaches, resulted in alignments that produced phylogenetic trees that are topologically and biologically

527 similar to those produced using a sequential approach that uses manual interventions. However,

528 the fully automated approaches were completed quickly as opposed to the numerous hours spent

529 on adjusting alignments for the traditional method. Although we used PASTA to generate the 
530 backbone for aligning the fragmentary data set with UPP, the results of our clade occupancy

531 comparisons between different alignment methods indicate that alignments from other software

532 will produce similar phylogenetic hypotheses for HBV. Researchers are encouraged to test

533 multiple alignment programs before choosing a backbone in which to align fragmentary data

534 against. Finally, phylogenetic trees estimated from both fully automated and traditional

535 alignments indicated most HBV genotypes are not monophyletic. This result suggests traditional

536 HBV genotype delineations should be reevaluated, and should encourage future studies to further

537 address this issue.

\section{Acknowledgments}

We are grateful for Brendan Morris, Massimo Pessino, and Pranjal Vachaspati for their

comments and contributions to this project. We also thank the University of Illinois Systematics

541 Group and in particular Sydney Cameron for helpful discussions and suggestions, and the staff at

542 Legends, Urbana, IL for technical support.

\section{Sources}

Arauz-Ruiz P, Norder H, Robertson BH, Magnius LO. 2002. Genotype H: a new Amerindian genotype of hepatitis B virus revealed in Central America. Journal of General Virology 83:2059-2073.

547 Arbizu C, Ruess H, Senalik D, Simon PW, Spooner DM. 2014. Phylogenomics of the carrot genus (Daucus, Apiaceae). American Journal of Botany 101: 1666-1685.

Beerenwinkel N, Gunthard HF, Roth V, Metzner KJ. 2012. Challenges and opportunities in estimating viral genetic diversity from next-generation sequencing data. Frontiers in Microbiology. 3(329)

Benson DA, Clark K, Karsch-Mizrachi I, Lipman DJ, Ostell J, Sayers EW. 2014. GenBank. Nucleic Acids Research. 42(D1): D32-D37.

Chen DS. 1993. From hepatitis to hepatoma: lessons from Type B viral hepatitis. Science 262:369-370.

Chooka, JB, Teob, WL, Ngeowb, YF, Teec, KK, Ngb, KP. Mohameda, R. 2015. Universal Primers for Detection and Sequencing of Hepatitis B Virus Genomes across Genotypes A to G. Journal of Clinical Microbiology 53(6):1831-1835.

Delsuc F, Brinkmann H, Philippe H. 2005. Phylogenomics and the reconstruction of the tree of life. Nature Reviews Genetics. 6: 361-375. 
559

560

561

562

563

564

565

566

567

568

569

570

571

572

573

574

575

576

577

578

579

580

581

582

583

584

585

586

587

588

589

590

591

592

593

594

595

596

597

598

599

600

601

602

603

604

605

606

607

608

Edgar RC, Batzoglou S. 2006. Multiple sequence alignment. Current Opinion in Structural Biology. 16: 368-373.

Edgar, RC. 2004. MUSCLE: multiple sequence alignment with high accuracy and high throughput RC Edgar. Nucleic Acids Research 32(5):1792-1797.

Eisen JA, Fraser CM. Phylogenomics: intersection of evolution and genomics. 2003. Science. 300: 17061707.

Faircloth, BC, Sorenson, L, Santini, F, Alfaro, ME. 2013. A phylogenomic perspective on the radiation of ray-finned fishes based on targeted sequencing of ultraconserved elements (UCEs). PLoS ONE 8:e65923.

Fast Tree-Comparison Tools. 2009. http://www.microbesonline.org/fasttree/treecmp.html. Last accessed October 10, 2017.

Galibert, F, Mandart, E, Fitoussi, F, Tiollais, P, Charnay, P. 1979. Nucleotide sequence of the hepatitis B virus genome (subtype ayw) cloned in E. coli. Nature 281:646-650. DOI:10.1038/281646a0.

Gouy, M, Guindon, S, Gascuel, O. 2010. SeaView version 4: a multiplatform graphical user interface for sequence alignment and phylogenetic tree building. Molecular Biology and Evolution 27:221-224.

Hall B. 2001. Phylogenetic Trees Made Easy A How-To Manual for Molecular Biologists. Sunderland: Sinaur Associates Inc.

Harkins KM, Schwartz RS, Cartwright RA, Stone AC. 2016. Phylogenomic reconstruction supports supercontinent origins for Leishmania. Infection, Genetics and Evolution. 38: 101-109.

Heath, TA, Hedtke, SM, Hillis, DM. 2008. Taxon sampling and the accuracy of phylogenetic analyses. Journal of Systematics and Evolution 46(3):239-257.

Hedtke, SM, Townsend, TM, Hillis, DM. 2006. Resolution of phylogenetic conflict in large data sets by increased taxon sampling. Systematic Biology 55(3):522-529.

Hernández, S, Venegas, M, Brahm, J, Villanueva, RA. 2014. Full-Genome Sequence of a Hepatitis B Virus Genotype F1b Clone from a Chronically Infected Chilean Patient. Genome Announcements 2(5):e01075-14. DOI:10.1128/genomeA.01075-14.

Heyduk K, Trapnell DW, Barrett CF, Leebens-Mack J. 2015. Phylogenomic analyses of species relationships in the genus Sabal (Arecaceae) using targeted sequence capture. Biological Journal of the Linnean Society. 117: 106-120.

Hillis DM, Moritz C, Mable BK. 1996. Molecular Systematics second edition. Sunderland: Sinauer Associates Inc.

Hossain KT, Patnaik D, Laxman S, Jain P, Bailey-Kellogg C, Ramakrishnan N. 2013. Improved multiple sequence alignments using coupled pattern mining. IEEE/ACM Transactions on Computational Biology and Bioinformatics. 10(5): 1098-1112.

Jarvis, ED, Mirarab, S, Aberer, AJ, Li, B, Houde, P, Li, C, Ho, SY, Faircloth, BC, Nabholz, B, Howard, JT, Suh, A, Weber, CC, da Ronseca, RR, Li, J, Zhang, F, Li, H, Zhou, L, Narula, N, Liu, L, Ganapathy, G, Bossau, B, Bayzid, MS, Zavidovych, G, Subramanian, S, Gabaldón T, CapellaGutiérrez, S, Huerta-Cepas, J, Rekepalli, B, Munch, K, Schierup, M, Lindow, B, Warren, WC, Ray, D, Green, RE, Bruford, MW, Zhan, X, Dixon, A, Li, S, Li, N, Huang, Y, Derryberry, EP, Bertelsen, MF, Sheldon, F, Brumfield, RT, Mello, C V, Lovell, PV, Wirthlin, M, Schneider, MPC, Prosdocimi, F, Samaniego, JA, Velazquez, AMV, Alfaro-Núñez, A, Campos, P, Petersen, B, Sicheritz-Ponten, T, Pas, A, Bailey, T, Scofield, P, Bunce, M, Lambert, DM, Zhou, Q, Perelman, P, Driskell, AC, Shapiro, B, Xiong, Z, Zeng, Y, Liu, S, Li, Z, Liu, B, \& Wu, K. 2014. Whole-genome analyses resolve early branches in the tree of life of modern birds. Science. 346: 1320-1331.

Jarvis ED. 2016. Perspectives from the avian phylogenomics project: questions that can be answered with sequencing all genomes of a vertebrate class. Annual Review of Animal Biosciences 4: 45-59.

Katoh, K, Misawa, K, Kuma, KI, Miyata, T. 2002. MAFFT: a novel method for rapid multiple sequence alignment based on fast Fourier transform. Nucleic Acids Research, 30(14), 3059-3066.

Katoh, K, Martin CF. 2012. Adding Unaligned Sequences into an Existing Alignment Using MAFFT and LAST. Bioinformatics 28(23):3144-3146 
60Xatoh K, Standley DM. 2013. MAFFT multiple sequence alignment software version 7: Improvements in performance 610 and usability. Molecular Biology and Evolution, 30(4):772-780.

611 Kemena C, Notredame C. 2009. Upcoming challenges for multiple sequence alignment methods in the 612 high-throughput era. Bioinformatics. 25: 2455-2465.

613 Kjer KM, Gillespie JJ, Ober KA. 2007. Opinions on multiple sequence alignment, and an empirical

614

615

616

617

618

comparison of repeatability and accuracy between POY and structural alignments. Syst Biol. 56:133146.

Kozlov AM, Aberer AJ, Stamatakis A. 2015. ExaML version 3: a tool for phylogenomic analyses on supercomputers. Bioinformatics. 31(15): 2577-2579.

Kurbanov F, Tanaka Y, Mizokami M. 2010. Geographical and genetic diversity of the human hepatitis B virus. Hepatology Research. 40(1):14-30.

Leache AD, Linkem CW. 2015. Phylogenomics of horned lizards (Genus: Phrynosoma) using targeted sequence capture data. Copeia. 103: 586-594.

Liaw, YF, Chu, CM. 2009. Hepatitis B virus infection. The Lancet 373:14-20.

Liu K, Linder CR, Warnow T. 2010. Multiple sequence alignment: a major challenge to large-scale phylogenetics. PLOS Currents. 2: RRN1198

Liu, K, Warnow, TJ, Holder, MT, Nelesen, SM, Alexandros, JY, Stamatakis, P, Linder, CR. 2012. SATéII: Very Fast and Accurate Simultaneous Estimation of Multiple Sequence Alignments and Phylogenetic Trees. Systematic Biology 61(1):90-106.

Lozano, R, Naghavi, M, Foreman, K, Lim, S, Shibuya, K, Aboyans, V, Abraham, J, Adair, T, Aggarwal, R, Ahn, SY, AlMazroa, MA, Alvarado, M, Anderson, HR, Anderson, LM, Andrews, KG, Atkinson, C, Baddour, LM, Barker-Collo, S, Bartels, DH, Bell, ML, Benjamin, EJ, Bennett, D, Bhalla, K, Bikbov, B, Abdulhak, AB, Birbeck, G, Blyth, F, Bolliger, I, Boufous, S, Bucello, C, Burch, M, Burney, P, Carapetis, J, Chen, H, Chou, D, Chugh, SS, Coffeng, LE, Colan, SD, Colquhoun, S, Colson, KE, Condon, J, Connor, MD, Cooper, LT, Corriere, M, Cortinovis, M, Courville de Vaccaro, K, Couser, W, Cowie, BC, Criqui, MH, Cross, M, Dabhadkar, KC, Dahodwala, N, Leo, DD, Degenhardt, L, Delossantos, A, Denenberg, J, Jarlais, DCD, Dharmaratne, SD, Dorsey, ER, Driscoll, T, Duber, H, Ebel, B, Erwin, PJ, Espindola, P, Ezzati, M, Feigin, V, Flaxman, AD, Forouzanfar, MH, Fowkes, FGR, Franklin, R, Fransen, M, Freeman, MK, Gabriel, SE, Gakidou, E, Gaspari, F, Gillum, RG, Gonzalez-Medina, D, Halasa, YA, Haring, D, Harrison, JE, Havmoeller, R, Hay, RJ, Hoen, B, Hotez, PJ, Hoy, D, Jacobsen, KH, James, SL, Jasrasaria, R, Jayaraman, S, Johns, N, Karthikeyan, G, Kassebaum, N, Keren, A, Khoo, J, Knowlton, LM, Kobusingye, O, Koranteng, A, Krishnamurthi, R, Lipnick, M, Lipshultz, SE, Ohno, SL, Mabweijano, J, MacIntyre, MF, Mallinger, L, March, L, Marks, GB, Marks, R, Matsumori, A, Matzopoulos, R, Mayosi, BM, McAnulty, JH, McDermott, MM, McGrath, J, Memish, ZA, Mensah, GA, Merriman, TR, Michaud, C, Miller, M, Miller, TR, Mock, C, Mocumbi, AO, Mokdad, AA, Moran, A, Mulholland, K, Nair, MN, Naldi, L, Narayan, KMV, Nasseri, K, Norman, P, O’Donnell, M, Omer, SB, Ortblad, K, Osborne, R, Ozgediz, D, Pahari, B, Pandian, JD, Rivero, AP, Padilla, RP, Perez-Ruiz, F, Perico, N, Phillips, D, Pierce, K, Pope III, CA, Porrini, E, Pourmalek, F, Raju, M, Ranganathan, D, Rehm, JT, Rein, DB, Remuzzi, G, Rivara, FP, Roberts, T, Rodriguez De León, F, Rosenfeld, LC, Rushton, L, Sacco, RL, Salomon, JA, Sampson, U, Sanman, E, Schwebel, DC, Segui-Gomez, M, Shepard, DS, Singh, D, Singleton, J, Sliwa, K, Smith, E, Steer, A, Taylor, JA, Thomas, B, Tleyjeh, IM, Towbin, JA, Truelsen, T, Undurraga, EA, Venketasubramanian, N, Vijayakumar, L, Vos, T, Wagner, GR, Wang, M, Wang, W, Watt, K, Weinstock, MA, Weintraub, R, Wilkinson, JD, Woolf, AD, Wulf, S, Yeh, P, Yip, P, Zabetian, A, Zheng, Z, Lopez, AD, Murray, CJL. 2012. Global and regional mortality from 235 cause of death for 20 age groups in 1990 and 2010: a systematic analysis for the Global Burden of Disease Study 2010. The Lancet. 380: 2096-2128.

Mashima J, Kodoma Y, Kosuge T, Fujisawa T, Katayama T, Nagasaki H, Okuda, Y, Kaminuma, E, Ogasawara, O, Okubo, K, Nakamura, Y, Takagi, T.. 2016. DNA data bank of Japan (DDBJ) progress report. Nucleic Acids Research. 44(D1): D51-D57. 
659

660

661

662

663

664

665

666

667

668

669

670

671

672

673

674

675

676

677

678

679

680

681

682

683

684

685

686

687

688

689

690

691

692

693

694

695

696

697

698

699

700

701

702

703

704

705

706

707

708

Mirarab S, Bayzid S, Boussau B, and Warnow T. 2014. Statistical binning enables an accurate coalescentbased estimation of the avian tree. Science 346 (6215) doi:10.1126/science.1250463

Mirarab S, Nguyen N, Guo S, Wang L-S, Kim J, Warnow T. 2015. PASTA: Ultra-Large Multiple Sequence Alignment for Nucleotide and Amino-Acid Sequences. Journal of Computational Biology. 22(5): 141230123051006. DOI:10.1089/cmb.2014.0156.

Misof B, Liu S, Meusemann K, Peters RS, Donath A, Mayer C, Frandsen, PB, Ware, J, Flouri, T, Beutel, RG, Niehuis, O, Petersen, M, Izquierdo-Carrasco, F, Wappler, T, Rust, J, Aberer, AJ, Aspöck, U, Aspöck, H, Bartel, D, Blanke, A, Berger, S, Böhm, A, Buckley, TR, Calcott, B, Chen, J, Friedrich, F, Fukui, M, Fujita, M, Greve, C, Grobe, P, Gu, G, Huang, Y, Jermiin, LA, Kawahara, AY, Krogmann, L, Kubiak, M, Lanfear, R, Letsch, H, Li, Y, Li, Z, Li, J, Lu, H, Machida, R, Mashimo, Y, Kapli, P, McKenna, DD, Meng, G, Nakagaki, Y, Navarrete-Heredia, JL, Ott, M, Ou, Y, Pass, G, Podsiadlowski, L, Pohl, H, von Reumont, BM, Schütte, K, Sekiya, K, Shimizu, S, Slipinski, A, Stamatakis, A, Song, W, Su, X, Szucsich, NU, Tan, M, Tan, X, Tang, M, Tang, J, Timelthaler, G, Tomizuka, S, Trautwein, M, Tong, X, Uchifune, T, Walzl, MG, Wiegmann, BM, Wilbrandt, J, Wipfler, B, Wong, TKF, Wu, Q, Wu, G, Xie, Y, Yang, S, Yang, Q, Yeates, DK, Yoshizawa, K, Zhang, Q, Zhang, R, Zhang, W, Zhang, Y, Zhao, J, Zhou, C, Zhou, L, Ziesmann, T, Zou, S, Li, Y, $\mathrm{Xu}$, X, Zhang, Y, Yang, H, Wang, J, Wang, J, Kjer, KM, Zhou, X.. 2014. Phylogenomics reveals the timing and pattern of insect evolution. Science. 346: 763-767.

Morrison DA. 2006. Multiple sequence alignment for phylogenetic purposes. Australian Systematic Botany. 19: 479-539.

Morrison DA. 2009. Why would phylogeneticists ignore computerized sequence alignment? Systematic Biology. 58: 150-158.

Nasir A, Caetano-Anolles G. 2015. A phylogenomic data-driven exploration of viral origins and evolution. Science Advances. 1:e1500527.

Nguyen N, Mirarab S, Kumar K, Warnow T. 2015. Ultra-large alignments using phylogeny-aware profiles. Genome Biology 16(1): 124. DOI:10.1186/s13059-015-0688-z.

Norder H, Courouce AM, Magnius LO. 1994. Complete genomes, phylogenetic relatedness, and structural proteins of six strains of the hepatitis B virus, four of which represent two new genotypes. Virology 198(2):489-503.

Okamoto H, Tsuda F, Sakugawa H, Sastrosoewignjo RI, Imai M, Miyakawa Y, Mayumi M. 1988. Typing hepatitis B virus by homology in nucleotide sequence: comparison of surface antigen subtypes. Journal of General Virology 69:2575-2583.

Osiowy, C, Giles, E, Tanaka, Y, Mizokami, M, Minuk, GY. 2006. Molecular evolution of hepatitis B virus over 25 years. Journal of Virology 80(21):10307-10314.

Ozaslan M, Ozaslan E, Barsgan A, Koruk, M. 2007. Mutations in the S gene region of hepatitis B virus genotype D in Turkish patients. Journal of Genetics 86(3):195-201.

Paradis E., Claude J., Strimmer K. 2004. APE: analyses of phylogenetics and evolution in R language. Bioinformatics 20:289-290. DOI:10.1093/bioinformatics/btg412

Philippe, H, Brinkmann, H, Lavrov, DV, Littlewood, TJ, Manuel, M, Worheide, G. 2011. Resolving difficult phylogenetic questions: why more sequences are not enough. PLoS Biology 9(3):e1000602.

Philippe H, Delcus F, Brinkmann H, Lartillot N. Phylogenomics. Annu Rev Ecol Evol Syst. 2005;36: 541-562.

Price MN, Dehal PS, Arkin AP. 2010. FastTree 2--approximately maximum-likelihood trees for large alignments. PLoS One. 5(3):e9490. DOI:10.1371/journal.pone.0009490.

Robinson DF, Foulds LR. 1981. Comparison of phylogenetic trees. Mathematical Biosciences. 53(12):131-147. DOI:10.1016/0025-5564(81)90043-2

Rokas A, Carroll SB. 2005. More Genes or More Taxa? The Relative Contribution of Gene Number and Taxon Number to Phylogenetic Accuracy. Molecular Biology and Evolution 22(5):1337-1344.

Rokas A, Williams BL, King N, Caroll SB. 2003. Genome-scale approaches to resolving incongruence in molecular phylogenies. Nature. 425: 798-804. 
709

710

711

712

713

714

715

716

717

718

719

720

721

722

723

724

725

726

727

728

729

730

731

732

733

734

735

736

737

738

739

740

741

742

743

744

745

746

747

748

749

750

751

752

753

754

755

756

757

758

759
Sanderson MJ, Driskell AC. 2003. The challenge of constructing large phylogenetic trees. Trends in Plant Sciences. 2003;8: 374-379.

Schmollinger, M, Nieselt, K, Kaufmann, M, Morgenstern, B. 2004. DIALIGN P: Fast pair-wise and multiple sequence alignment using parallel processors BMC Bioinformatics 5:128.

Shi, W, Zhang, Z, Ling, C, Zheng, W, Zhu, C, Carr, MJ, Higgins, DG. 2013. Hepatitis B virus subgenotyping: History, effects of recombination, misclassifications, and corrections. Infection, Genetics and Evolution 16:355-361.

Shi, Y-H. 2012. Correlation between hepatitis B virus genotypes and clinical outcomes. Japanese Journal of Infectious Disease 65:476-482.

Sievers F, Wilm, A., Dineen, D, Gibson, TJ, KSarplus, K, Li, W, Lopez, R, McWilliam, H, Remmert, M, Söding, J, Thompson, JD, Higgins, DG. 2011. Fast, scalable generation of high-quality protein multiple sequence alignments using Clustal Omega. Molecular Systems Biology 7(539). doi:10.1038/msb.2011.75.

Simmonds P, Midgley S. 2005. Recombination in the genesis and evolution of hepatitis B virus genotypes. Journal of Virology 79:15467-76.

Soltis DE, Gitzendanner MA, Soltis PS. 2007. A 567-taxon data set for angiosperms: the challenges posed by Bayesian analyses of large data sets. International Journal of Plant Sciences. 168: 137-157.

Sonnhammer, ELL, Gabaldon, T, Sousa da Silva, AW, Martin, M, Robinson-Rechavi, M, Boeckmann, B, Thomas, PD, Dessimoz, C. 2014. Big data and other challenges in the quest for orthologs. Bioinformatics 30(21):2993-2998.

Stamatakis, A. 2014. RAxML Version 8: A tool for Phylogenetic Analysis and Post-Analysis of Large Phylogenies. Bioinformatics 30(9):1312-1313.

Stuyver L, De Gendt S, Van Geyt C, Zoulim F, Fried M, Schinazi RF, Rossau R. 2000. A new genotype of hepatitis B virus: complete genome and phylogenetic relatedness. Journal of General Virology 81(Pt 1):67-74.

Subramanian, AR, Weyer-Menkhoff, J, Kaufmann, M, Morgenstern, B. 2005. DIALIGN-T: An improved algorithm for segment-based multiple sequence alignment. BMC Bioinformatics 6:66.

Sukumaran, J, Holder, MT. 2010. DendroPy: A Python library for phylogenetic computing. Bioinformatics 26:1569-1571.

Tatematsu K, Tanaka Y, Kurbanov F, Sugauchi, F, Mano, S, Maeshiro, T, Nakayoshi, T, Wakuta, M, Miyakawa, Y, Mizokami, M. 2009. A genetic variant of hepatitis B virus divergent from known human and ape genotypes isolated from a Japanese patient and provisionally assigned to new genotype J. Journal of Virology 83:10538-47.

Tran TT, Trinh TN, Abe K. 2008. New complex recombinant genotype of hepatitis B virus identified in Vietnam. Journal of Virology 82:5657-63.

Weber, B. 2005. Genetic variability of the S gene of hepatitis B virus: clinical and diagnostic impact. Journal of Clinical Virology 32:102-112.

Wu, G, Ding, H, Zeng, C. 2008. Overview of HBV whole genome data in public repositories and the Chinese HBV reference sequences. Progress in Natural Science 18:13-20.

Yang, Z. 1998. On the best evolutionary rate for phylogenetic analysis. Systematic Biology 47(1):125133.

Yates A, Akanni W, Amode MR, Barrell D, Billis K, Carvalho-Silva D, Cummins, C, Clapham, P, Fitzgerald, S, Gil, L, Giron, CG, Gordon, L, Hourlier, T, Hunt, SE, Janacek, SH, Johnson, N, Juettemann, T, Keenan, S, Lavidas, I, Martin, FJ, Maurel, T, McLaren, W, Murphy, DN, Nag, R, Nuhn, M, Parker, A, Patricio, M, Pignatelli, M, Rahtz, M, Riat, HS, Sheppard, D, Taylor, K, Thormann, A, Vullo, A, Wilder, SP, Zadissa, A, Birney, E, Harrow, J, Muffato, M, Perry, E, Ruffier, M, Spudich, G, Trevanion, SJ, Cunningham, F, Aken, BL, Zerbino, DR, Flicek, P.. Ensembl 2016. Nucleic Acids Res. 2016;44: D710-D716 doi:10.1093/nar/gkv1157.

Ye, Y, Cheung, DWL, Wang, Y, Yiu, SM, Zhang, Q, Lam, TW, Ting, HF. 2015. GLProbs: Aligning multiple sequences adaptively. IEEE/ACM Transactions on Computational Biology and Bioinformatics 12(1):67-78. 
760 Figures

761

762 Figure 1. Genetic map of the hepatitis B virus genome. Arrows indicate the reading frames of 763 genes.

764 Figure 2. Workflow outline for the alignment of hepatitis B virus genome and total (genomes + 765 fragments) data sets. Additional details for each step are illustrated in Supplementary Fig. 1.

766

767

768

769

770

771

772

773

774

775

776

777

778

779

780

781

782

783

784

Figure 3. Heatmaps representing the pairwise comparisons of hepatitis B virus phylogenies. A)

Ratio of incompatible edges to total edges in a comparison. B) Robinson-Foulds distances

between two phylogenies. Darker cells indicate two trees with greater differences. Alignments used to estimate the phylogenies are indicated on the $\mathrm{x}$ - and $\mathrm{y}$-axes..

Figure 4. Box-and-whisker plots showing the distributions of pairwise comparison values for hepatitis B virus phylogenies. (A) and (B) show the ratio of incompatible edges to total edges in a comparison. (C) and (D) show Robinson-Foulds distances. Alignments used to estimate the phylogenies are indicated on the x-axis. (A) and (C) are colored according to the edge collapse threshold (bootstrap value). (B) and (D) are colored according to the second tree in a comparison.

Figure 5. Hepatitis B virus genotype occupancy. A) Histograms showing the proportion of each genotype that makes up the minimum clade including all individuals of that genotype. Genotypes are indicated on the x-axis, and genotype occupancy is shown as percentages along the y-axis. Bootstrap support collapse thresholds are indicated above each panel. B) Fully bifurcating (i.e., no branches collapsed), midpoint-rooted cladogram of HBV sequences from the PASTA genome alignment. Tips are colored according to genotype.

Supplementary Figure 1. Workflow undertaken to align publicly available hepatitis B virus sequences using a traditional approach (automated alignment followed by manual adjustments). 
785 Genome alignment (top) and total (fragmentary + genomes; bottom) data sets are both illustrated.

786 Blue boxes represent processes (i.e. specific analyses), and green shapes represent data products

787 (e.g. files).

788 Supplementary Figure 2. Pairwise RF distances between each ML tree estimated from the

789 PASTA, Muscle, Muscle-Manual, Clustal Omega, and MAFFT genome alignments, after

790 pruning edges with less than 50\% support. The results show that PASTA, Clustal Omega and

791 MAFFT result in similar tree topology for highly supported edges.

792 Supplementary Figure 3. Distribution of the pairwise p-distances between each sequence for

793 the PASTA alignment (top), Manual alignment (middle), and S-region alignment (bottom). The

794 alignments show similar pairwise p-distance distributions.

795

796

797 


\section{Table $\mathbf{1}$ (on next page)}

Comparison of different alignment approaches used for the genome and total (genomes + fragments) data sets from HBV. 


\begin{tabular}{|c|c|c|}
\hline Method & Genomes Only & All Sequences \\
\hline Traditional & MUSCLE + manual & MUSCLE + manual \\
\hline Automated & MUSCLE & UPP: Genomes-manual backbone \\
\hline Automated & PASTA & UPP: PASTA backbone \\
\hline
\end{tabular}

1 


\section{Table 2 (on next page)}

Comparison of alignment methods.

All alignments were attempted using Genomes_Manual_degapped.fasta as the input file. 


\begin{tabular}{llll} 
Method & Alignment length & Wall clock time & Method reference \\
\hline Manual & 4269 & & NA \\
PASTA & 3423 & 3.5 hours & Mirarab et al. 2014 \\
MAFFT & 4578 & 4 min & Mafft 7.305b; Katoh and Standley 2013 \\
MUSCLE & 4938 & 32 hours & Muscle v3.8.31; Edgar 2004 \\
Clustal Omega & 3846 & 2.25 hours & Clustal Omega 1.2.4; Sievers et al. 2011 \\
\hline
\end{tabular}

1 
Figure 1

Genetic map of the hepatitis B virus genome.

Arrows indicate the reading frames of genes.

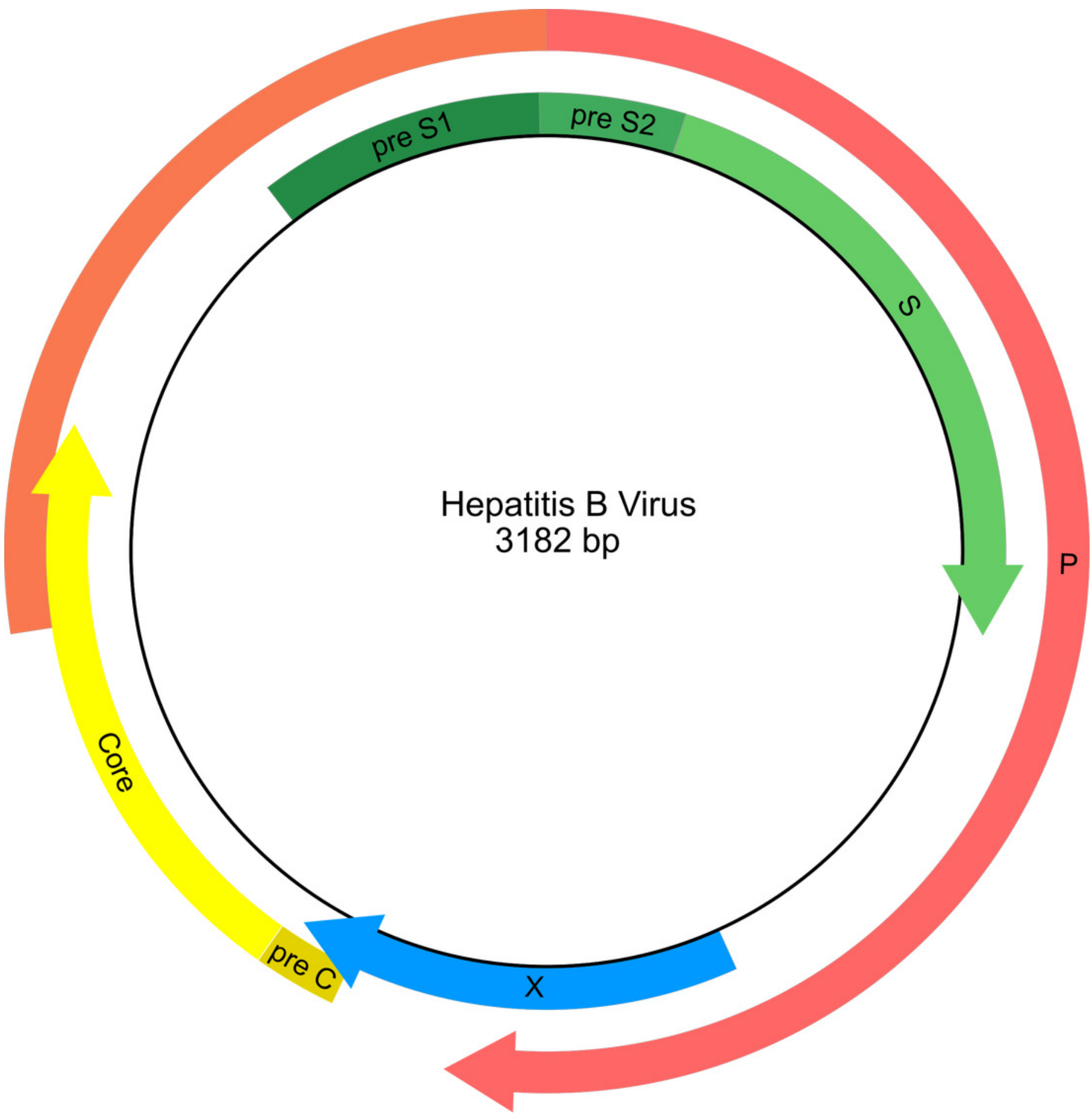




\section{Figure 2 (on next page)}

Workflow outline for the alignment of hepatitis B virus genome and total (genomes + fragments) data set.

Additional details for each step are illustrated in Supplementary Fig. 1. 


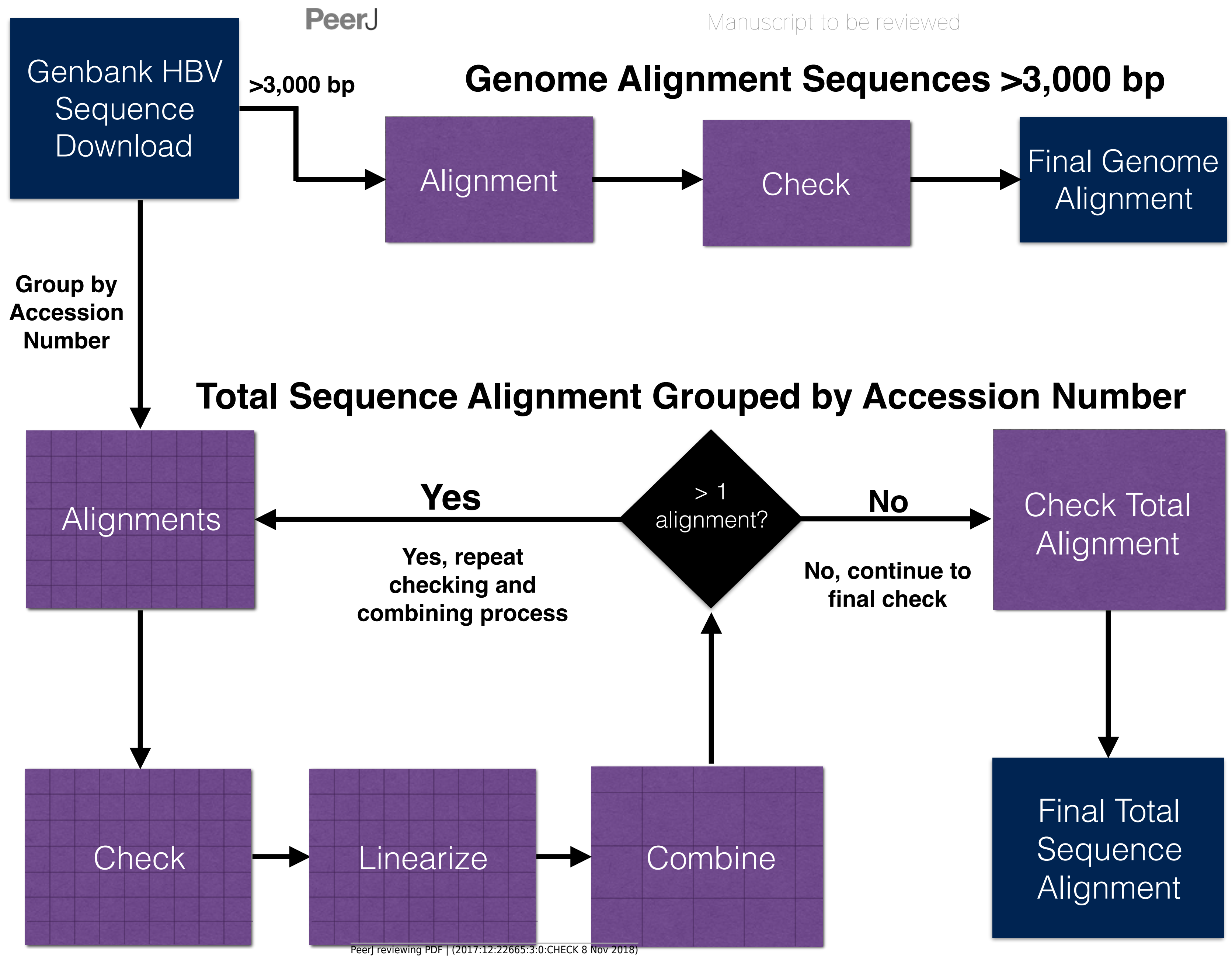




\section{Figure 3 (on next page)}

Heatmaps representing the pairwise comparisons of hepatitis B virus phylogenies.

A) Ratio of incompatible edges to total edges in a comparison. B) Robinson-Foulds distances between two phylogenies. Darker cells indicate two trees with greater differences. Alignments used to estimate the phylogenies are indicated on the $x$ - and $y$-axes. 
Ratio of Incompatible Edge to Total Edges

Total UPP PASTA

Total UPP manual

Total manua

S-region

Genomes PASTA

Genomes MUSCLE

Genomes manual

Total UPP PASTA

Total UPP manua

Total manual

S-region

Genomes PASTA

Genomes MUSCLE

Genomes manual

ષ્ષ

Total UPP PASTA

Total UPP manual

Total manual

S-region

Genomes PASTA

Genomes MUSCLE

Genomes manual

Total UPP PASTA

Total UPP manua

Total manual

S-region

Genomes PASTA

Genomes MUSCLE

Genomes manual

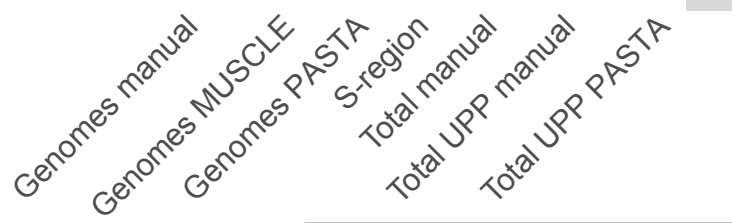

Peer-reviewing PDF | (2017:12:22665:3:0:CHECK 8 Nov 2018)
Robinson-Foulds Distance

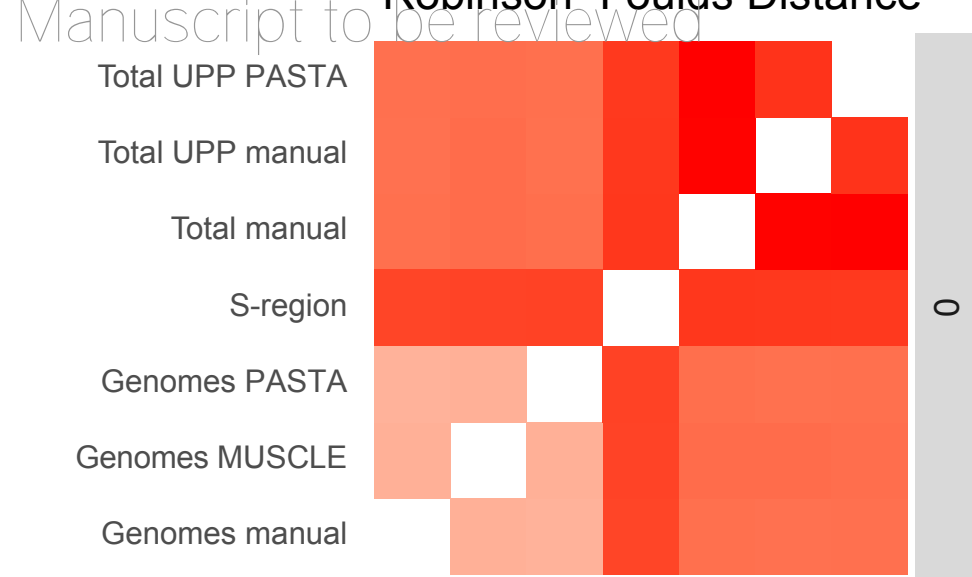

Total UPP PASTA

Total UPP manual

Total manual

S-region

Genomes PASTA
Incompatibility ratio

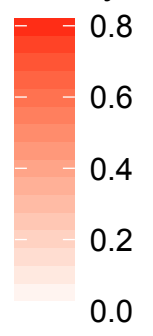

जे

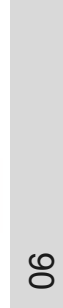

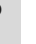

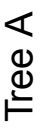

Genomes MUSCLE

Genomes manual

Total UPP PASTA

Total UPP manual

Total manual

S-region

Genomes PASTA

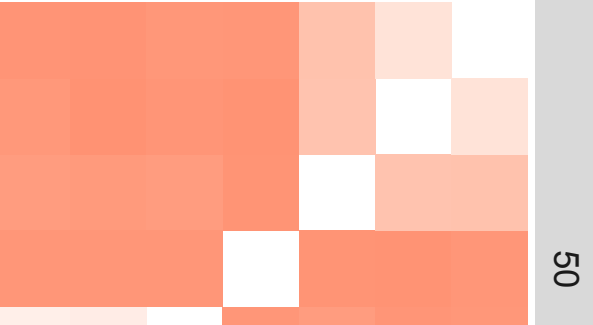

Genomes MUSCLE

Genomes manual

Total UPP PASTA

Total UPP manual

Total manual

S-region

Genomes PASTA

Genomes MUSCLE

Genomes manual

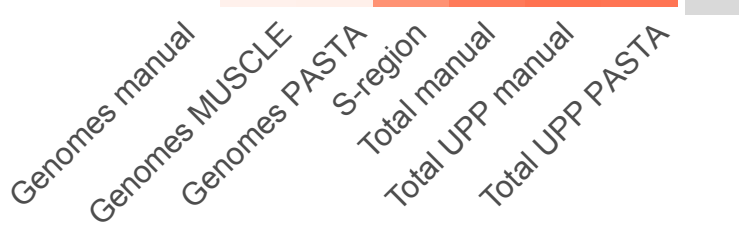

Tree B 


\section{Figure 4(on next page)}

Box-and-whisker plots showing the distributions of pairwise comparison values for hepatitis $B$ virus phylogenies.

(A) and (B) show the ratio of incompatible edges to total edges in a comparison. (C) and (D) show Robinson-Foulds distances. Alignments used to estimate the phylogenies are indicated on the x-axis. (A) and (C) are colored according to the edge collapse threshold (bootstrap value). (B) and (D) are colored according to the second tree in a comparison. 


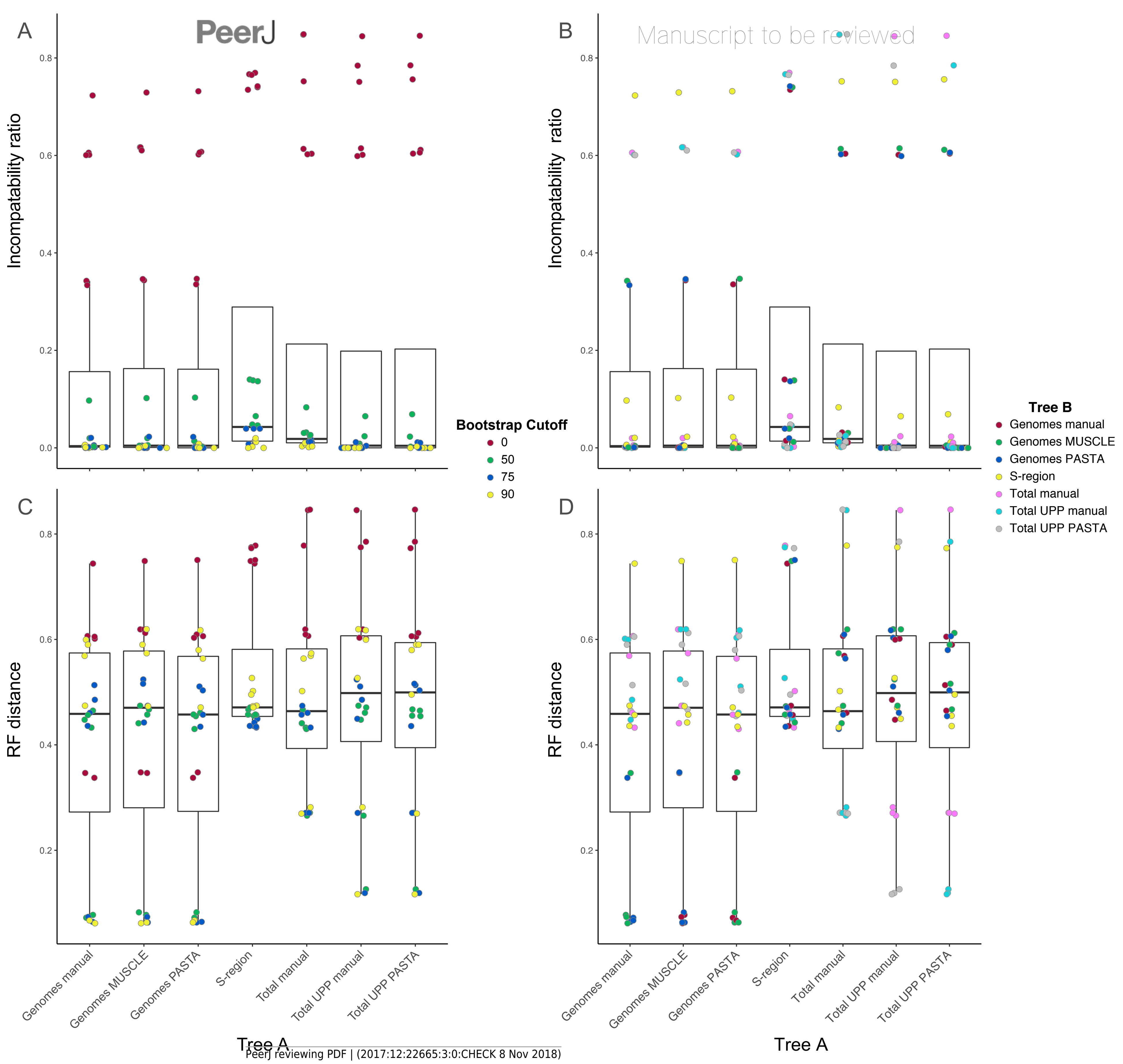


Figure $\mathbf{5}$ (on next page)

Hepatitis B virus genotype occupancy.

A) Histograms showing the proportion of each genotype that makes up the minimum clade including all individuals of that genotype. Genotypes are indicated on the x-axis, and genotype occupancy is shown as percentages along the y-axis. Bootstrap support collapse thresholds are indicated above each panel. B) Fully bifurcating (i.e., no branches collapsed), midpoint-rooted cladogram of HBV sequences from the PASTA genome alignment. Tips are colored according to genotype. 
A
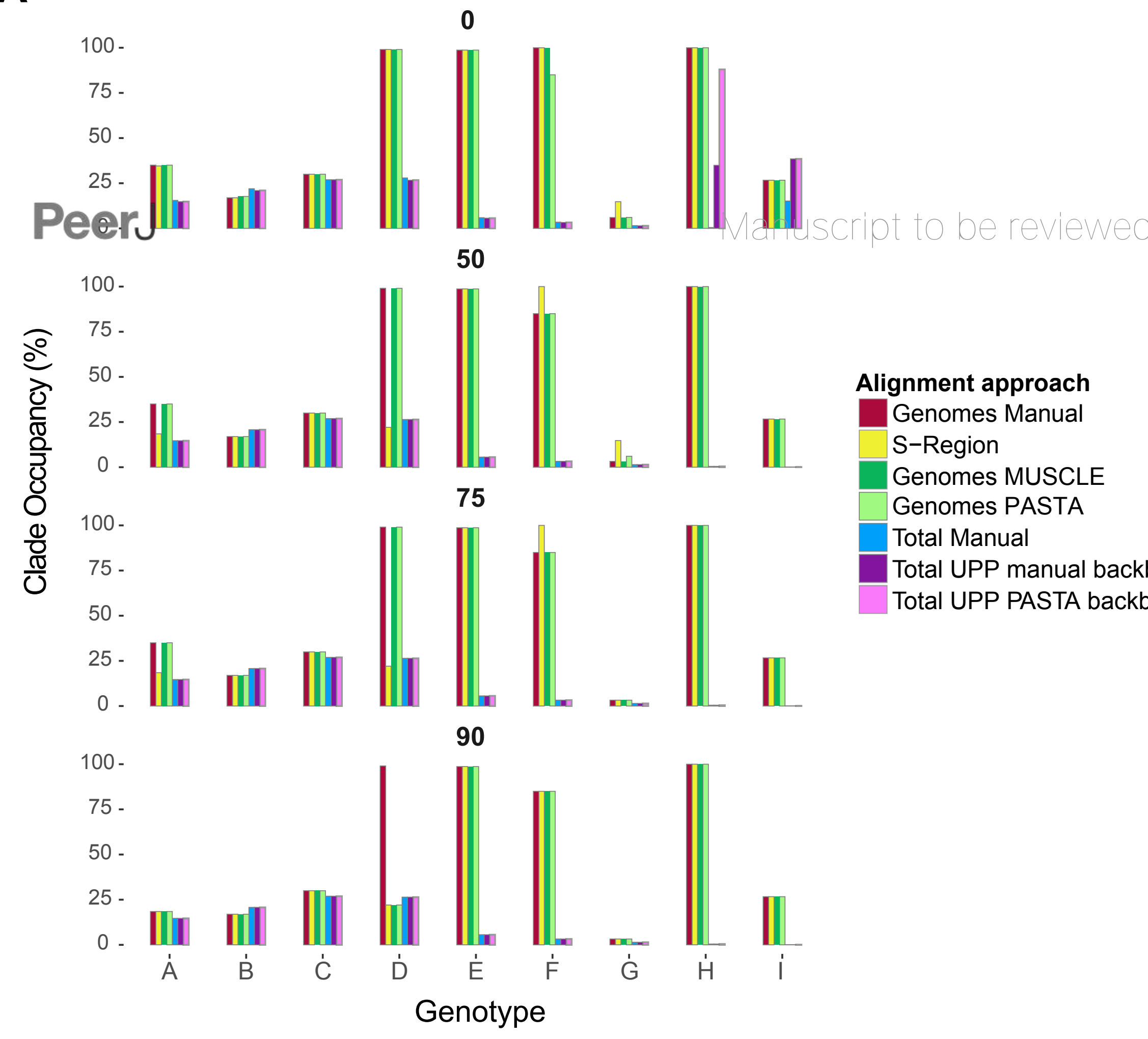

B

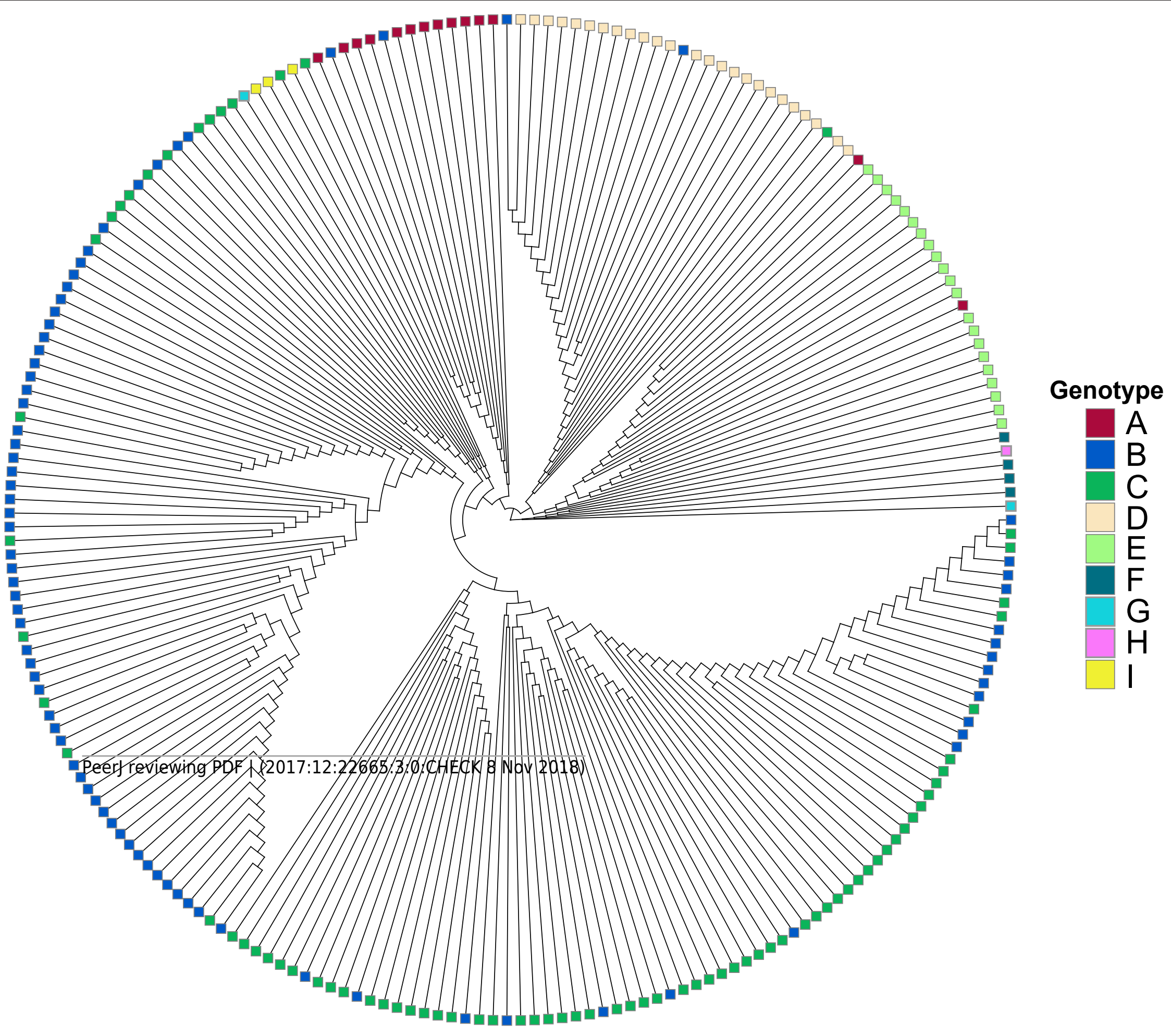

